\title{
A Note on the Stability of Predictor-Corrector Techniques*
}

\author{
By James Case
}

\begin{abstract}
The theory of schlicht mappings is used to estimate stepsizes which guarantee the stability of a linear multistep method for the integration of ordinary differential equations.
\end{abstract}

In this paper we shall consider the propagated error which arises in the approximate solution of the initial value problem

$$
\dot{x}=A x, \quad x\left(t_{0}\right)=x_{0},
$$

where $A$ is an $N \times N$ constant real matrix, by the general linear $k$-step finite difference equation

$$
a_{k} y(n+k)+\cdots+a_{0} y(n)=h\left(b_{k} \dot{y}(n+k)+\cdots+b_{0} \dot{y}(n)\right) .
$$

We shall show that, as in the case of a single equation, the stability of the method (2) depends on the moduli of the zeros of a certain polynomial, and that if the eigenvalues of $A$ have negative real parts, the stepsize $h$ may be chosen so small that all the zeros lie inside the unit circle. We then give a table of the numerical constants needed for choosing the $h$ in such fashion, and exhibit the results of some experiments designed to illustrate the importance of the effects under consideration.

I. The Statement of the Theorem. In order to give our theorem as simple a form as possible, it is helpful to introduce a certain amount of terminology. Most of the terms which follow either are, or closely resemble terms, which are in general use. Notation:

(a) Let $\lambda_{1}, \cdots, \lambda_{N}$ be the eigenvalues of $A$, and let $\phi_{1}, \cdots, \phi_{N}$ be their respective arguments, which we shall take to lie in the interval $0 \leqq \phi<2 \pi$. Also, let $\lambda=$ $\max _{i}\left|\lambda_{i}\right|$ and $\theta=\max _{i}\left|\pi-\phi_{i}\right|$, where $i=1,2, \cdots, N$.

(b) Let

$$
\begin{aligned}
& \rho(z)=a_{k} z^{k}+\cdots+a_{1} z+a_{0}=a_{k}\left(z-\alpha_{1}\right) \cdots\left(z-\alpha_{k}\right) \neq 0, \\
& \sigma(z)=b_{k} z^{k}+\cdots+b_{1} z+b_{0}=b_{k}\left(z-\beta_{1}\right) \cdots\left(z-\beta_{k}\right) .
\end{aligned}
$$

We assume that $\alpha_{1}=1$, and that $\alpha_{2}, \cdots, \alpha_{k}$ lie inside the unit circle.

(c) In addition, we assume $\rho^{\prime}(1)=\sigma(1)$, and that $\rho(z)$ and $\sigma(z)$ have no common zeros.

(d) Let the pair $(\rho, \sigma)$ be called a method. Then each method and choice of the constant $h$, called the stepsize, defines a unique finite difference Eq. (2). Assumptions (b) and (c) now guarantee that the methods $(\rho, \sigma)$ are stable and consistent in the sense of Dahlquist [2].

Received December 11, 1968, revised February 11, 1969.

* Sponsored by the Mathematics Research Center, United States Army, Madison, Wisconsin, under Contract No. DA-31-124-ARO-D-462. 
(e) Let $C$ be a fixed contour lying inside the unit circle, enclosing $\alpha_{2}, \cdots, \alpha_{k}$, and let $Q=\min _{z} \in_{C}|\rho(z) / \sigma(z)|$.

(f) Let $r_{\theta}$ be defined for $0 \leqq \theta \leqq \pi / 2$ as the least positive root of the equation

$$
\frac{Q r}{(1-r)^{2}}+2 \cos \left(\pi+\theta+\log \frac{1+r}{1-r}\right)=0 \text {. }
$$

Values of $r_{\theta}$ for several choices of the arguments $Q, \theta$ are found in Table 1 at the end of the paper.

(g) Let $x(t)$ be the exact solution of the problem (1). We will abuse this notation to the extent of writing $x(n)=x\left(t_{0}+n h\right)$.

(h) Let $y(0)=x(0)$, and let $y(1), \cdots, y(k-1)$ be $k-1$ real $N$-vectors called starting values for the Eq. (2).

(i) Let $y(n)$ be the approximation for $x(n)$ obtained by use of (2) and the starting values $y(0), \cdots, y(k-1)$. As usual we shall assume that the method is used iteratively, until convergence is achieved so that the sequence $\{y(n)\}$ depends only on the corrector formula (2) and not on the choice of the predictor formula.

(j) Let $\epsilon(n)=x(n)-y(n)$. We call $\epsilon(n)$ the error at the $n$th step.

Next we shall define two different kinds of stability. The first is entirely standard, and requires no explanation. The second is a weakening of the concept of $A$-stability introduced by Dahlquist [3]. It is of interest because methods of arbitrarily high order may be $B$-stable, while only methods of order two or less may be $A$-stable.

Definition 1. The problem (1) will be called asymptotically stable if $\lim _{t \rightarrow \infty}|x(t)|=$ 0 for every choice of the initial value $x_{0}$.

Definition 2 . The method $(\rho, \sigma)$ will be called $B$-stable with respect to the problem (1) if for some $\mu>0$ the zeros of the polynomials

$$
\rho(z)-h \lambda_{i} \sigma(z), \quad i=1,2, \cdots, N,
$$

called the characteristic polynomials of the method $(\rho, \sigma)$ lie on or inside the unit circle whenever $|h \lambda|<\mu$. Thus we may demonstrate that a given method $(\rho, \sigma)$ is $B$-stable with respect to a particular problem by showing that the zeros of the polynomials (5) are of less than unit modulus for sufficiently small stepsizes $h$. In contrast, a method is $A$-stable only if, when applied to an appropriate problem, the zeros of (5) remain in the unit circle for every positive $h$. It is in this sense that $B$-stability is a weakening of Dahlquist's $A$-stability.

We may now state the following:

THEOREM. The method $(\rho, \sigma)$, which is stable and consistent because of assumptions (a) and (b), is B-stable with respect to the problem (1) if and only if (1) is asymptotically stable. In addition, $\mu$ may be taken to be $r_{\theta} Q$.

II. The Characteristic Polynomials. To begin with, let us introduce the finitedifference operator $E$ defined for every vector valued function $v$ on the positive integers by the relation $E v(n)=v(n+1)$. We may now write Eq. (2) in the shorter form

$$
\rho(E) y(n)=h \sigma(E) \dot{y}(n) .
$$

The principal value of this notation arises from the easily verified fact that the 
operators $p(E)$, where $p$ is any polynomial, commute with ordinary matrix multiplication.

If we now consider the sequence $x(n)$ defined previously, we observe that it satisfies the relation

$$
\rho(E) x(n)=h \sigma(E) \dot{x}(n)+T(n)=h \sigma(E) A x(n)+T(n),
$$

while the sequence $y(n)$ satisfies

$$
\rho(E) y(n)=h \sigma(E) \dot{y}(n)-r(n)=h \sigma(E) A y(n)-r(n) .
$$

The terms $r(n)$ and $T(n)$ are called the round-off and truncation errors at the $n$th step respectively. Subtracting (7) from (6), and making the substitution $\epsilon(n)=$ $x(n)-y(n)$, we see that the error $\epsilon(n)$ at the $n$th step satisfies

$$
\rho(E) \epsilon(n)=h \sigma(E) A \epsilon(n)+T(n)+r(n) .
$$

Let us now assume that $r(n)=r$ and $T(n)=T$ are constant, and set $A=P^{-1} B P$, where $P$ is a constant nonsingular matrix and $B$ will be explained later.

If we multiply Eq. (8) by $P$, and set $P \epsilon(n)=\eta(n)$ wherever it appears, we have

$$
\rho(E) \eta(n)-h \sigma(E) B \eta(n)=\varepsilon
$$

where $\varepsilon=P(r+T)$ and we need henceforth discuss only the normalized error $\eta(n)$, from which $\epsilon(n)$ may be computed. In particular, if $P$ may be chosen so that $B$ is diagonal, the vector Eq. (9) yields the following $N$ scalar equations for the components of $\eta(n)$ :

$$
\left(\rho(E)-h \lambda_{i} \sigma(E)\right) \eta_{i}(n)=\varepsilon_{i} \quad i=1, \cdots, N .
$$

It is well known that if the zeros of the polynomials (5) are distinct, and if none of the eigenvalues $\lambda_{i}$ is equal to zero, the solutions of (10) are

$$
\eta_{i}(n)=A_{i 1} \zeta_{i 1}^{n}+\cdots+A_{i k} \zeta_{i k}^{n}-\varepsilon_{i} / h \lambda_{i} \sigma(1)
$$

$\zeta_{i l}, \cdots, \zeta_{i k}$ being the zeros of the $i$ th polynomial (5). If the zeros are not distinct, or if some $\lambda_{i}$ should vanish, the right side of the Eq. (11) must be replaced by an alternate expression, which, however retains the property that its nonconstant terms all approach zero as $n$ grows large, provided only that the zeros are of less than unit modulus. In view of this uniformity of behaviour, we shall not pause to discuss the various forms which Eq. (11) may take, but will proceed directly to the study of the behaviour of the zeros of the polynomials (5).

III. The Proof of the Theorem. To begin with, let us observe that if $|t|<Q$ Rouché's theorem guarantees that the polynomial $\rho(z)-t \sigma(z)$ has just one zero outside the contour $C$. We shall denote it by $\zeta_{1}(t)$ and call it the principal zero of $\rho-t \sigma$. The other zeros shall be called extraneous. If $t \neq 0$, then $\zeta_{1}(t)$ is not a zero of either $\rho$ or $\sigma$. We choose a point $t_{0}$ of the disc $|t|<Q$, and set $z_{0}=\zeta_{1}\left(t_{0}\right)$. Then the representation

$$
\rho(z)-t_{0} \sigma(z)=\sum_{j=1}^{k} a_{j}\left(z-z_{0}\right)^{j}
$$


together with the fact that $z_{0}$ is a simple zero of $\rho-t_{0} \sigma$, yield the fact that $a_{1}=$ $\rho^{\prime}\left(z_{0}\right)-t_{0} \sigma^{\prime}\left(z_{0}\right) \neq 0$. But since $t_{0}=\rho\left(z_{0}\right) / \sigma\left(z_{0}\right)$, we have $\sigma\left(z_{0}\right) \rho^{\prime}\left(z_{0}\right)-\rho\left(z_{0}\right) \sigma^{\prime}\left(z_{0}\right) \neq$ 0 , which implies that the function $t=\rho(z) / \sigma(z)$ has a nonvanishing derivative at $z=z_{0}$, and possess a unique analytic inverse $z=f(t)$ in some neighborhood of $t_{0}$. Moreover, since $\rho(f(t))-t \sigma(f(t))=0, f(t)$ can only be $\zeta_{1}(t)$, which is therefore analytic and univalent near $t_{0}$, and hence throughout the disc $|t|<Q$.

A function which is analytic and univalent on a disc, however, is subject to strict regularity conditions. In particular, (see Hayman [4, p. 4 and p. 139]) if $f(z)$ is analytic and univalent in $|z|<1, f(0)=0, f^{\prime}(0)=1$, (that is, if $f$ is a schlicht mapping) we have the inequalities

$$
|f(z)| \leqq r /(1-r)^{2} \text { and }|\arg (f(z) / z)| \leqq \log ((1+r) /(1-r))
$$

for all $|z| \leqq r<1$. So now let us set $\tau=t / Q$ and define an auxiliary function $\varphi(\tau)$ on $|\tau|<1$ by the relation $\varphi(\tau)=\left(\zeta_{1}(t)-1\right) / Q$. Then $\varphi(\tau)$ is analytic and univalent on the unit disc, and $\varphi(0)=0$. Moreover, the Maclaurin expansion $\zeta_{1}(t)=1+$ $t+\cdots$ (see Henrici [5, p. 237]) shows that $\varphi^{\prime}(0)=1$. Thus we have the inequalities

$$
\begin{aligned}
\frac{1}{Q}\left|\zeta_{1}(t)-1\right| & =|\varphi(\tau)| \leqq r /(1-r)^{2} \\
\left|\arg \left(\zeta_{1}(t)-1\right)-\arg t\right| & =|\arg (\varphi(\tau) / \tau)| \leqq \log ((1+r) /(1-r))
\end{aligned}
$$

whenever $|t| \leqq r Q<Q$.

Now let us suppose that $\tau$ is constrained to lie in the sector $|\tau|<r,|\pi-\arg \tau|$ $\leqq \theta<\pi / 2$. By the inequalities (14), the image of this sector under the schlicht map $w=Q \varphi(\tau)$ is contained in the domain bounded by the circular arc $|w|=Q r /(1+r)^{2}$ and the two curves $|\pi-\arg w|=\theta+\log ((1+r) /(1-r))$. But if

$$
\frac{Q r}{(1-r)^{2}} \leqq-2 \cos \left(\pi+\theta+\log \frac{1+r}{1-r}\right)
$$

or equivalently if $r \leqq r_{\theta}, w=Q \varphi(\tau)$ will surely lie in the circle $|w+1| \leqq 1$ whose equation is $|w| \leqq-2 \cos (\arg w), \pi / 2<\arg w<3 \pi / 2$. A similar argument shows that if $-\pi / 2<\arg w<\pi / 2, \varphi(\tau)$ must lie in the right-half plane, for small $|\tau|$, and hence cannot lie in $|w+1| \leqq 1$. Finally, if $\tau$ is pure imaginary, we need only observe that the curvature of the graph of $\log ((1+r) /(1-r))$ is zero when $r=0$ to conclude that $Q \varphi(\tau)$ must lie outside $|w+1| \leqq 1$ for small $|\tau|$ in this case too. Thus, since $\left|\zeta_{1}(t)\right| \leqq 1$ if and only if $w=Q \varphi(\tau)$ lies in the circle $|w+1| \leqq 1$, and since the extraneous zeros are all of less than unit modulus so long as $|t|<Q$, we have shown that all of the zeros of $\rho-t \sigma$ must lie in the unit circle if $\pi / 2<$ $\arg t=\theta<3 \pi / 2$, and $|t| \leqq r_{\theta} Q$, and further, that if $-\pi / 2 \leqq \arg t=\theta \leqq \pi / 2$, at least one of the zeros must lie outside $|z|<1$ for small values of $|t|$. The theorem follows by allowing $t$ to take on the $N$ values $h \lambda_{i}$, and recalling the fact that (1) is asymptotically stable if and only if $\operatorname{Re} \lambda_{i}<0$ for $i=1,2, \cdots, N$.

IV. Remarks. In the next section, we will consider some possible applications of the theorem and give the results of a few experiments designed to establish its importance. Before we pass on to such a discussion, however, we should observe that, in part II, we assumed the matrix $A$ to be diagonalizable, and so arrived at 
the Eq. (10) for the components $\eta_{i}(n)$ of the normalized error $\eta(n)$. If such is not the case, however, we may assume $B$ to be in Jordan canonical form, so that (10) is replaced by

$$
\rho(E)-h \lambda_{i} \sigma(E) \eta_{i}(n)=h \sigma(E) \eta_{i+1}(n)+\varepsilon_{i}
$$

where $\eta_{i+1}(n)$ may be regarded as known. The general solution of $\left(10^{\prime}\right)$ is the sum of a particular solution plus the general solution of the related homogeneous equation, which is already known. Now if $\eta_{i+1}(n)=B_{i 1} \zeta_{i 1}^{n}+\cdots+B_{i k} \zeta_{i k}^{n}+K$ where $K$ is a constant, then the right side $R_{i}(n)$ of $\left(10^{\prime}\right)$ is

$$
\begin{aligned}
R_{i}(n) & =\varepsilon_{i}+h \sigma(E) \eta_{i+1}(n)=h \sigma(E)\left(B_{i 1} \zeta_{i 1}^{n}+\cdots+B_{i k} \zeta_{i k}^{n}+K\right) \\
& =C_{i 1} \zeta_{i 1}^{n}+\cdots+C_{i k} \zeta_{i k}^{n}+\varepsilon_{i}{ }^{\prime}
\end{aligned}
$$

where $C_{i j}=h B_{i j} \sigma\left(\zeta_{j}\right)$ and $\varepsilon_{i}{ }^{\prime}=\varepsilon_{i}+h K \sigma(1)$. But a particular solution of $\left(10^{\prime}\right)$ is given by the expression

$$
z_{i}(n)=\sum_{m=k}^{n} R_{i}(m) y_{m}(n)
$$

where the sequence $\left\{y_{m}(n)\right\}$ are certain particular solutions of the related homogeneous equation. Hence each $y_{m}(n)$ has the form

$$
y_{m}(n)=A_{1}^{m} \zeta_{i 1}+\cdots+A_{k}{ }^{m} \zeta_{i k}^{n} .
$$

It may now be shown by a rather lengthy but obvious calculation, that $z_{i}(n)$, and hence every solution of $\left(10^{\prime}\right)$, is the sum of a sequence which is bounded and one which tends to zero with the $n$th powers of the zeros of the polynomials (5), so long as these are of less than unit modulus. That is to say, the behaviour of the sequence $\eta(n)$, and hence $\epsilon(n)$, is essentially the same whether or not $A$ is diagonalizable, and is at least qualitatively the same as the behaviour of the error which arises in case $N=1$. This we offer by way of justification of our definition of $B$-stability. If further justification is still in order, we suggest that it should come from empirical evidence that the distinction it makes is indeed a useful one.

V. Numerical Results and Applications. In order to demonstrate the importance of $B$-stability to the numerical solution of systems (1), we wrote a program to solve the system

$$
\left(\begin{array}{l}
\dot{x} \\
\dot{y}
\end{array}\right)=\left(\begin{array}{cc}
0 & 1 \\
-a b & a+b
\end{array}\right)\left(\begin{array}{l}
x \\
y
\end{array}\right)+\left(\begin{array}{c}
0 \\
f(t)
\end{array}\right)
$$

the initial conditions being chosen so that the transient terms in the solution would vanish. The eigenvalues of this system are $a$ and $b$. The program used single precision arithmetic, was supplied with exact starting values, and made use of only a single prediction and a single correction at each step. It was hoped that although so rudimentary a program would be totally unsatisfactory in the general case, it would still be possible to obtain good answers for the stable problems. In general this hope was realized. The characteristic polynomial for such an algorithm may be shown to be (see Chase [1])

$$
\rho(z)-h^{2} \lambda_{i}{ }^{2} b_{k} \mu(z)-h \lambda_{i} \nu(z)-h \sigma^{*}(z)
$$


where $(\rho, \sigma)$ is the correction method, $(\nu, \mu)$ is the prediction method, and $\sigma^{*}$ is the polynomial obtained by subtracting $b_{k} z^{k}$ from $\sigma(z)$. If the eigenvalues are constrained to be real, it is possible to test (20) numerically to find the interval $Q_{1} \leqq h \lambda_{i} \leqq 0$ for which all of the zeros remain inside of the unit circle. In this way it was possible to determine exactly for which stepsizes the methods were $B$-stable, and for which one or more of the characteristic zeros lay outside of the unit circle.

The predictor used was an eight point Newton-Cotes formula, and the correctors were respectively, the Adams 7-point formula, and the formulae $W_{7}(.4)$, and $R_{7}(.4)$, of Hull and Newbery [7]. $f(t)$ was set equal to $t^{2} \sin t+t^{3}$, the eigenvalues $(a, b)$ were chosen successively to be $(1,2),(-1,2),(-1,-2),(3,4),(-3,4)$, and $(-3,-4)$, and the stepsizes of $2^{-1}, 2^{-2}, \cdots, 2^{-9}$ were tried. As might be expected, the answers were largely meaningless. In fact it was only for the pairs $(-1,-2)$ and $(-3,-4)$ that the analytic solutions ever exceeded the errors, but for these two problems, the answers were quite good. To be more precise, we plotted the maximum error over the interval $0 \leqq t \leqq 10$ against $b h$, where $h$ is the stepsize and $b$ is the larger eigenvalue, the result always being a curve of the form:

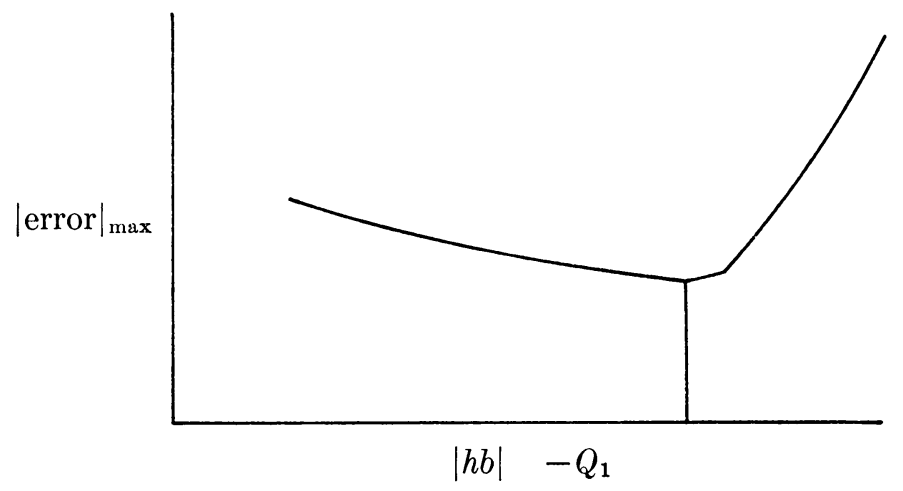

Here $Q_{1}$ is the value of $h b$ for which one of the zeros of (20) first escapes from the unit circle. To the left of $Q_{1}$, the errors were of the order $10^{-3}$ to $10^{-5}$, while those to the right varied between $10^{3}$ and $10^{20}$. In other words, since the correct answers were of the order $10^{2}$, the answers gave from five to seven significant digits for stable values of $h b$, and none at all for unstable ones. In the case of the unstable systems, the pictures had the same general shape, but the answers were never good enough to yield even one significant digit. Tables 2, 3 and 4 list the quantitative results of applying the Adams method, $W_{7}(.4)$ and $R_{7}(.4)$ respectively to the six problems mentioned, and constitute, we believe, rather striking evidence of the effect of $B$-stability on the results of numerical integration.

Finally, let us note that our results bear on a wider range of problems than they have been stated for. If we wish to integrate

$$
\dot{x}=f(x, t) ; \quad x\left(t_{0}\right)=x_{0}
$$

where $f \in C^{1}$ for $t \geqq t_{0}$, we may still write Eqs. (6) and (7) in the form

$$
\begin{aligned}
& \rho(E) x(n)=h \sigma(E) \dot{x}(n)+T(n) \\
& \rho(E) y(n)=h \sigma(E) \dot{y}(n)-r(n)
\end{aligned}
$$

and observe that $\epsilon_{i}(n)=x_{i}(n)-y_{i}(n)=f_{i}\left(x(n), t_{n}\right)-f_{i}\left(y(n), t_{n}\right)=\nabla f_{i}\left(z_{i}\right) \cdot \epsilon(n)$ 
so that $\epsilon(n)=A \epsilon(n)$, where $z_{i}$ is some point on the line segment joining $x(n)$ and $y(n)$, and $A$ is the matrix whose rows are the vectors $\nabla f_{i}\left(z_{i}\right)$. Hence Eq. (8) may be written just as before, and making the assumptions that $A, r(n)$ and $T(n)$ are constant, we can proceed again as in Section II. Thus we may extend our analysis to nonlinear problems for which the eigenvalues of the variational matrix $A$ are sufficiently well known, and we may construct programs which monitor the stepsize $h$ in order to keep the zeros of the characteristic polynomials always within the unit circle. When such an approach is feasible, it will often result in considerably reduced computing times, due to the larger values of $h$ which it permits.

\begin{tabular}{|c|c|c|c|}
\hline \multicolumn{4}{|c|}{ TABLE 1} \\
\hline $180 \theta / \pi$ & $r_{\theta}$ & $180 \theta / \pi$ & $r_{\theta}$ \\
\hline $\begin{array}{l}15^{\circ} \\
30^{\circ} \\
40^{\circ} \\
50^{\circ} \\
60^{\circ}\end{array}$ & $\begin{array}{l}.5708 \\
.4762 \\
.4063 \\
.3313 \\
.2522\end{array}$ & $\begin{array}{l}70^{\circ} \\
75^{\circ} \\
85^{\circ} \\
87^{\circ} \\
89^{\circ}\end{array}$ & $\begin{array}{l}.1697 \\
.1277 \\
.0426 \\
.0255 \\
.0085\end{array}$ \\
\hline \multicolumn{4}{|c|}{$Q=.2$} \\
\hline $180 \theta / \pi$ & $r_{\theta}$ & $180 \theta / \pi$ & $r_{\theta}$ \\
\hline $\begin{array}{l}15^{\circ} \\
30^{\circ} \\
40^{\circ} \\
50^{\circ} \\
60^{\circ}\end{array}$ & $\begin{array}{l}.5669 \\
.4720 \\
.4020 \\
.3272 \\
.2485\end{array}$ & $\begin{array}{l}70^{\circ} \\
75^{\circ} \\
85^{\circ} \\
87^{\circ} \\
89^{\circ}\end{array}$ & $\begin{array}{l}.1668 \\
.1252 \\
.0416 \\
.0250 \\
.0083\end{array}$ \\
\hline \multicolumn{4}{|c|}{$Q=.3$} \\
\hline $180 \theta / \pi$ & $r_{\theta}$ & $180 \theta / \pi$ & $r_{\theta}$ \\
\hline $\begin{array}{l}15^{\circ} \\
30^{\circ} \\
40^{\circ} \\
50^{\circ} \\
60^{\circ}\end{array}$ & $\begin{array}{l}.5630 \\
.4678 \\
.3978 \\
.3232 \\
.2448\end{array}$ & $\begin{array}{l}70^{\circ} \\
75^{\circ} \\
85^{\circ} \\
87^{\circ} \\
89^{\circ}\end{array}$ & $\begin{array}{l}.1639 \\
.1229 \\
.0407 \\
.0244 \\
.0812\end{array}$ \\
\hline \multicolumn{4}{|c|}{$Q=.5$} \\
\hline $180 \theta / \pi$ & $r_{\theta}$ & $180 \theta / \pi$ & $r_{\theta}$ \\
\hline $\begin{array}{l}15^{\circ} \\
30^{\circ} \\
40^{\circ} \\
50^{\circ} \\
60^{\circ}\end{array}$ & $\begin{array}{l}.5551 \\
.4594 \\
.3894 \\
.3151 \\
.2377\end{array}$ & $\begin{array}{l}70^{\circ} \\
75^{\circ} \\
85^{\circ} \\
87^{\circ} \\
89^{\circ}\end{array}$ & $\begin{array}{l}.1584 \\
.1185 \\
.0390 \\
.0233 \\
.0077\end{array}$ \\
\hline \multicolumn{4}{|c|}{$Q=.7$} \\
\hline $180 \theta / \pi$ & $r_{\theta}$ & $180 \theta / \pi$ & $r_{\theta}$ \\
\hline $\begin{array}{l}15^{\circ} \\
30^{\circ} \\
40^{\circ} \\
50^{\circ} \\
60^{\circ}\end{array}$ & $\begin{array}{l}.5472 \\
.4510 \\
.3811 \\
.3073 \\
.2308\end{array}$ & $\begin{array}{l}70^{\circ} \\
75^{\circ} \\
85^{\circ} \\
87^{\circ} \\
89^{\circ}\end{array}$ & $\begin{array}{l}.1531 \\
.1143 \\
.0375 \\
.0224 \\
.0074\end{array}$ \\
\hline
\end{tabular}




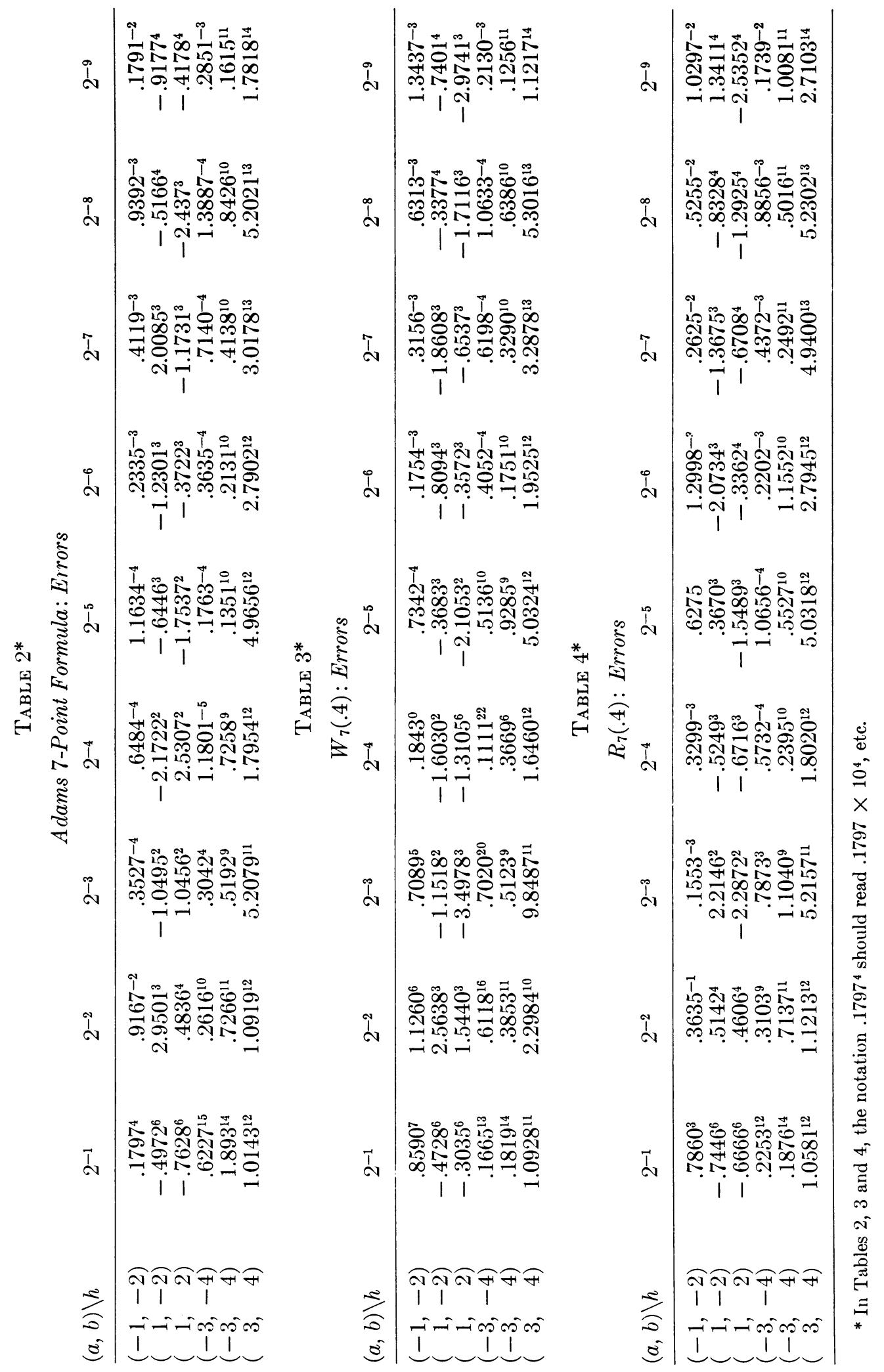


University of Wisconsin

Mathematics Research Center

Madison, Wisconsin 53706

1. P. E. Chase, "Stability properties of predictor-corrector methods for ordinary differential equations," J. Assoc. Comput. Mach., v. 9, 1962, pp. 457-468. MR 29 \#738.

2. G. G. DAHLQUIST, "Stability and error bounds in numerical integration of ordinary differential equations," Kungl. Tekn. Högsk. Handl. Stockholm., v. 130, 1959, 87 pp. MR 21 \#1706.

3. G. G. DAHLQUist, "A special stability problem for linear multistep methods," Nordisk Tidskr. Informations-Behandling, v. 3, 1963, pp. 27-43. MR 30 \#715.

4. W. K. Hayman, Multivalent Functions, Cambridge Tracts in Math. and Math. Phys., no. 48, Cambridge Univ. Press, New York, 1958. MR 21 \#7302.

5. P. Henrici, Discrete Variable Methods in Ordinary Differential Equations, Wiley, New York, 1962. MR 24 \#B1772.

6. P. Henrici, Error Propagation for Difference Methods, Wiley, New York, 1963. MR 27 \#4365.

7. T. E. Hull \& A. C. R. Newbery, "Integration procedures which minimize propagated errors," J. Soc. Indust. Appl. Math., v. 9, 1961, pp. 31-47. MR 22 \#11519.

8. T. E. Hull \& A. C. R. NEW BERY, "Corrector formulas for multi-step integration methods," J. Soc. Indust. Appl. Math., v. 10, 1962, pp. 351-369. MR 27 \#2130. 